\title{
Correlação entre o desenvolvimento neuropsicomotor e a continência urinária em crianças - Revisão sistemática
}

\author{
Review of the correlation between neuropsychomotor \\ development and the urinary continence in children
}

Resumo O conceito de desenvolvimento neuropsicomotor (DNPM) se refere ao aumento da capacidade do indivíduo na realização de funções cada vez mais complexas, num processo sequencial e ordenado. Não existe uma medida quantitativa para o DNPM, porém, pode-se especificar níveis e graus de desenvolvimento por meio de escalas específicas. A ansiedade de pais ou cuidadores em desfraldar uma criança que não se encontra no período de desenvolvimento adequado para esse tipo de trabalho pode causar sofrimento para os mesmos e para as crianças e ser causa de disfunções do trato urinário. $\mathrm{O}$ objetivo deste estudo foi apontar estudos que discutem a correlação entre o DNPM ao estado de continência urinária em crianças. Foi realizada uma busca bibliográfica na base de dados Lilacs, PubMed e SciELO, sendo examinados, ao todo, 52 artigos. Parte das referências foi utilizada de livros que abordaram assuntos compatíveis com o estudo. Concluiu-se que o DNPM e seus marcadores específicos podem servir de variável importante para uma marcação mais acurada, ou ao menos vir a somar insights quanto ao desenvolvimento em paralelos dos controles motor e miccional. Ao considerar o controle miccional dependente de musculatura estriada esquelética, como o rabdosfíncter, parece logicamente provável que o controle esfincteriano esteja diretamente correlacionado ao DNPM. Entretanto, sugere-se estudos para correlacionar o DNPM da criança com a continência urinária, visando iniciar a discussão quanto à definição de marcadores mais precisos de desenvolvimento para uma orientação mais adequada quanto ao início do treinamento esfincteriano.

Palavras-chave: Desenvolvimento infantil. Micção. Sistema URINÁRIO.

Bianca da Silva Januário ABDalla ${ }^{1}$

DHAIANY GoMES DouRado ERICA Feio Carneiro Nunes ${ }^{I I}$ Gustavo Fernando Sutter LATORRE ${ }^{\text {III }}$

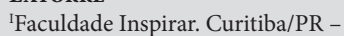
Brasil

"Universidade do Estado do Pará.

Belém/PA - Brasil

"IIRede Perineo.net. Florianópolis/ SC - Brasil
Abstract The concept of neuropsychomotor development (DNPM) refers to the increase in the individual's ability to perform increasingly complex functions, in a sequential and orderly process. There is no quantitative measure for the DNPM, but it is possible to specify levels and degrees of development through specific scales. The anxiety of parents or caregivers to unfurl a child who is not in the appropriate development period for this type of work can cause suffering for them and for children and be a cause of urinary tract disorders. The aim of this study was to point out studies that discuss the correlation between DNPM and urinary continence status in children. A bibliographic se- 
arch was performed in the Lilacs, PubMed and SciELO database, and 52 articles were examined. Part of the references was used from books that addressed subjects compatible with the study. It was concluded that the DNPM and its specific markers can serve as an important variable for more accurate marking, or at least add to the development of motor and voiding controls in parallel. When considering voiding control dependent on skeletal striated musculature, such as the rhabdosphincter, it seems logically likely that sphincter control is directly correlated to DNPM. However, studies are suggested to correlate the child's DNPM with urinary continence, aiming to start the discussion on the definition of more accurate developmental markers for a more adequate guidance regarding the beginning of sphincter training.

Keywords: Child Development. Urination. Urinary Tract.

\section{INTRODUÇÃO}

O conceito clássico de desenvolvimento neuropsicomotor (DNPM) se refere ao aumento da capacidade do indivíduo na realização de funções cada vez mais complexas, num processo sequencial e ordenado. ${ }^{1}$ O DNPM é determinado pela combinação entre os fatores genéticos e ambientais, influências e experiências do passado e situação do momento, de modo que as tarefas propostas para as crianças pelos seus pais e pela escola têm grande influência na aquisição de novas habilidades. ${ }^{2,3}$

Pode acontecer de a criança manifestar em seu desenvolvimento motor características de outra idade que não aquela na qual ela se encontra. Isso irá variar de um indivíduo para o outro. A aquisição das habilidades não está ligada direta e intrinsecamente ao tempo, e sim ao processo de desenvolvimento que é singular para cada ser humano, mas a ideia clássica de que as mudanças são ordenadas em sequência não é excluída. Os caminhos podem variar de um estado a outro, algumas etapas podem não ser atingidas ou plenamente estabelecidas, mas a sequência não deixa de existir. ${ }^{3}$
A observação do DNPM pode ser realizada por meio de escalas específicas. Estas se baseiam no amadurecimento percebido pela aquisição de novas habilidades ao longo do tempo, o que pode ser observado e acompanhado, porém não pode ser medido com a precisão desejável. Não existe uma medida quantitativa para o desenvolvimento, porém pode-se especificar níveis e graus de desenvolvimento em termos de amadurecimento. ${ }^{4}$

$O$ fato da não existência de um instrumento padronizado dificulta essa avaliação, tornando-a quase inexequível. Isto contribui para que, não raras vezes, alterações no desenvolvimento passem despercebidas, de modo a se tornar evidentes muito tarde, quando a criança ingressa no Ensino Fundamental. ${ }^{4}$

Entre os marcos do desenvolvimento infantil está o controle esfincteriano, que constitui um desafio para os pais e para as crianças. É um dos primeiros eventos para que a criança se torne autossuficiente, iniciando ativamente por volta dos dois anos de idade. Todas as crianças, exceto as que tiveram algum distúrbio neurológico, irão adquirir esse controle, mas muitas poderão apresentar problemas durante esse desen- 
volvimento, culminando em incontinência urinária ou enurese noturna. Todavia, poucas questões na área do desenvolvimento infantil envolvem maior preocupação do que as áreas referentes ao treinamento de esfíncteres e os seus distúrbios. Além disso, crianças incontinentes apresentam problemas de autoestima. ${ }^{5,6}$

A criança é considerada com controle esfincteriano, ou continente, a partir do momento em que é capaz de ter consciência de sua própria necessidade de eliminar a urina e as fezes, podendo iniciar o ato sem um lembrete ou um preparo por parte dos pais ou cuidadores. Não é incomum que pais ou cuidadores apresentem expectativas precoces em relação à idade ideal para o início desse treinamento. ${ }^{5}$

A ansiedade de pais ou cuidadores em desfraldar uma criança que ainda não se encontra no período de desenvolvimento adequado para esse tipo de trabalho, pode causar sofrimento nos mesmos e, especialmente, para essas crianças. ${ }^{6} \mathrm{O}$ conhecimento, portanto, de marcadores adequados do desenvolvimento, pode minimizar esse tipo de sofrimento. Sabe-se, por exemplo, que o controle evacuatório é alcançado em média por volta dos dois anos de idade, enquanto o controle miccional diurno acontece por volta dos quatro anos de idade e o noturno por volta dos seis, variando ainda entre meninos e meninas. ${ }^{6}$

Apesar de o controle esfincteriano fazer parte do desenvolvimento normal das crianças, não há parâmetros descritos na literatura que correlacionem as fases do DNPM com o desenvolvimento do contro- le miccional e evacuatório. Assim, o presente estudo tem como objetivo correlacionar o desenvolvimento neuropsicomotor da criança com o estado da continência urinária, visando iniciar a discussão quanto à definição de marcadores mais precisos de desenvolvimento para uma orientação mais adequada quanto ao início do treinamento esfincteriano, evitando distúrbios miccionais futuros decorrentes do início precoce ou tardio do processo de desfralde.

\section{Metodologia}

Trata-se de uma revisão sistemática da literatura, para a qual foram efetuadas, no período de novembro de 2017 a janeiro de 2018, buscas nas bases de dados Lilacs, PubMed e SciELO. Utilizaram-se as palavras-chave: desenvolvimento neuropsicomotor, treinamento esfincteriano, crianças e micção e suas variantes em inglês: Child development, Toilet training, children e urination segundo os descritores em ciências da saúde (DeCS).

Foram incluídos ensaios clínicos nos idiomas inglês, português, espanhol e francês, publicados nos anos de 1995 a 2015, que correlacionassem o desenvolvimento neuropsicomotor (DNPM) e o treinamento esfincteriano (TE) em crianças com idade pré-escolar. Foram excluídos estudos em cobaias e estudos de revisão.

A seleção dos estudos se deu pela escolha de artigos pelo título, em seguida pelo resumo, e por fim pela leitura na íntegra. $\mathrm{O}$ fluxo de estudos por meio do processo de seleção é apresentado na Figura 1. 


\section{Artigos identificados por meio das bases de dados $(n=105)$}

\section{Pesquisas adicionais identificadas por outros meios $(n=0)$}

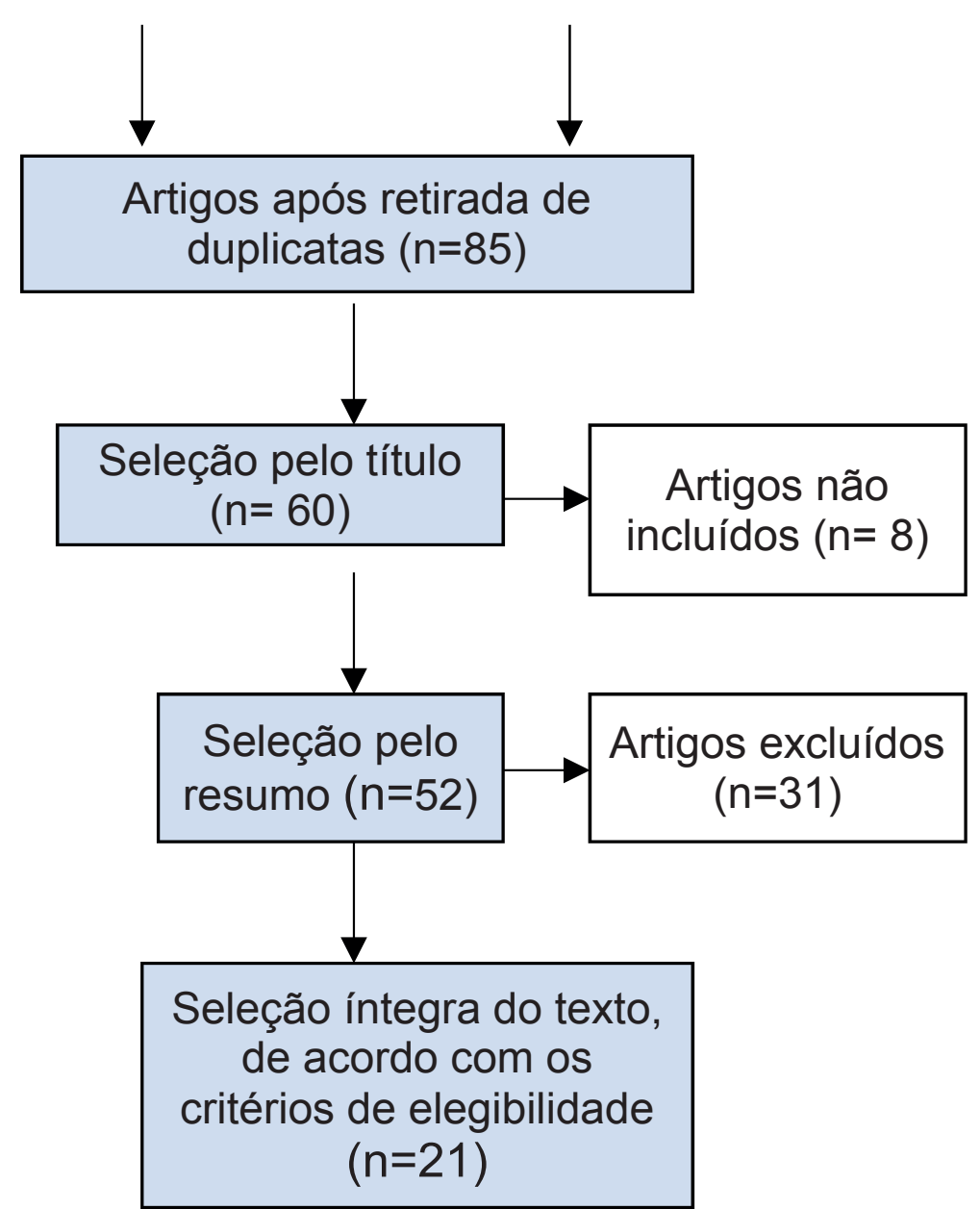

Figura 1: Seleção dos estudos para inclusão na revisão sistemática

\section{RESUlTADOS}

Foram analisados, na íntegra, 52 artigos, porém somente 21 artigos fizeram parte dos resultados. A Tabela 1 relaciona esses artigos que abordaram como tema principal o desenvolvimento neuropsicomotor e o treinamento esfincteriano (TE) de crianças. 


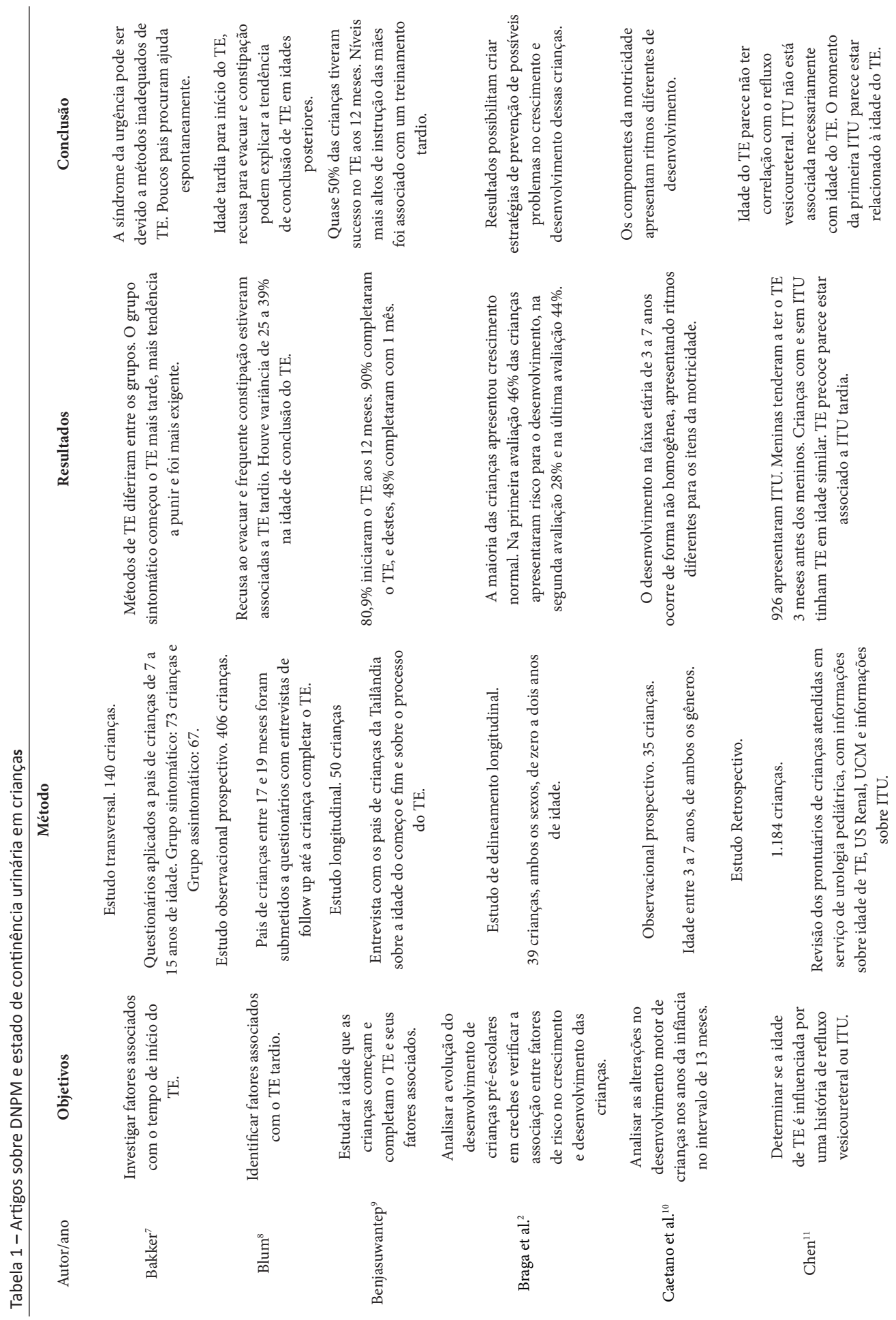



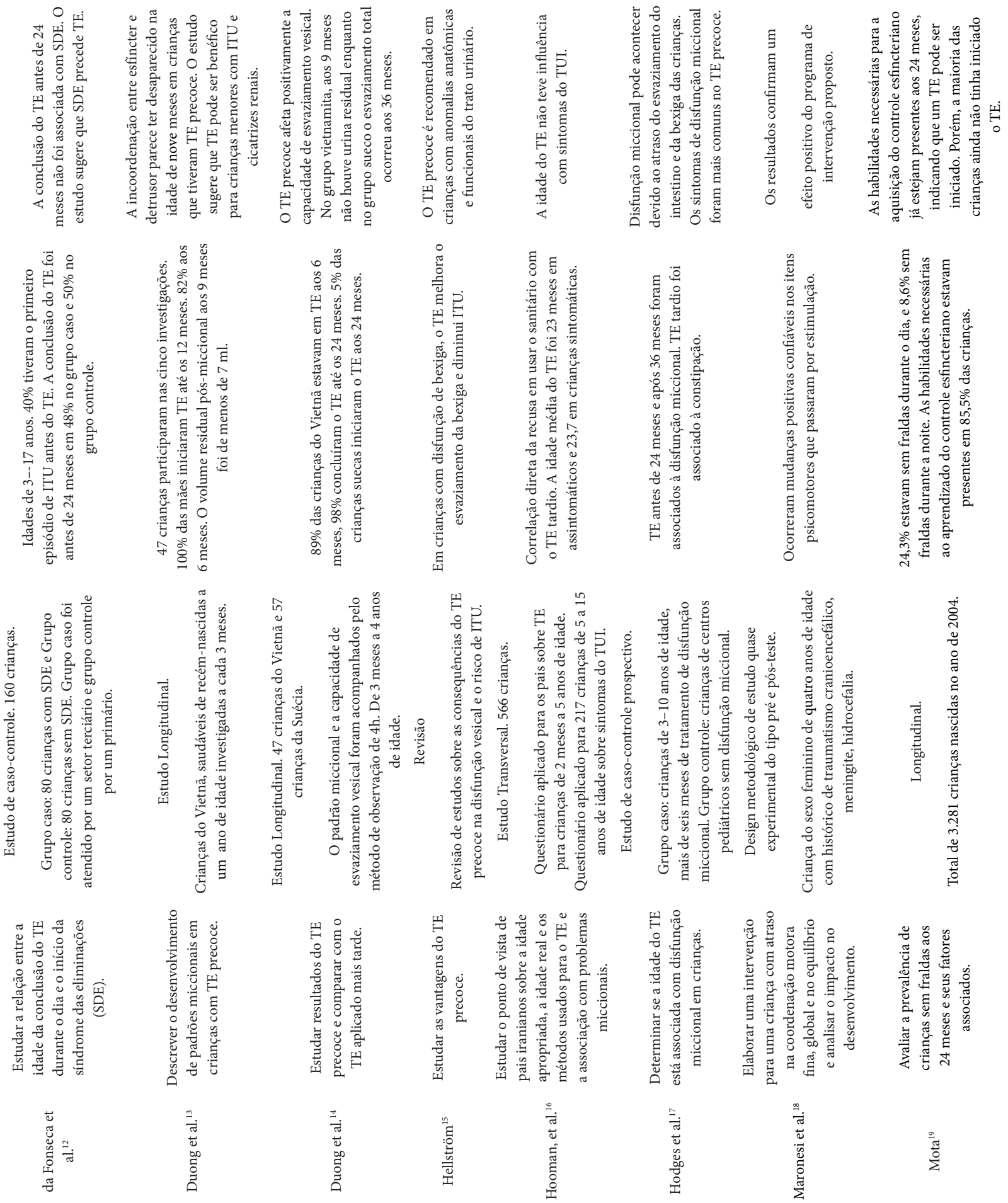

हैँ 

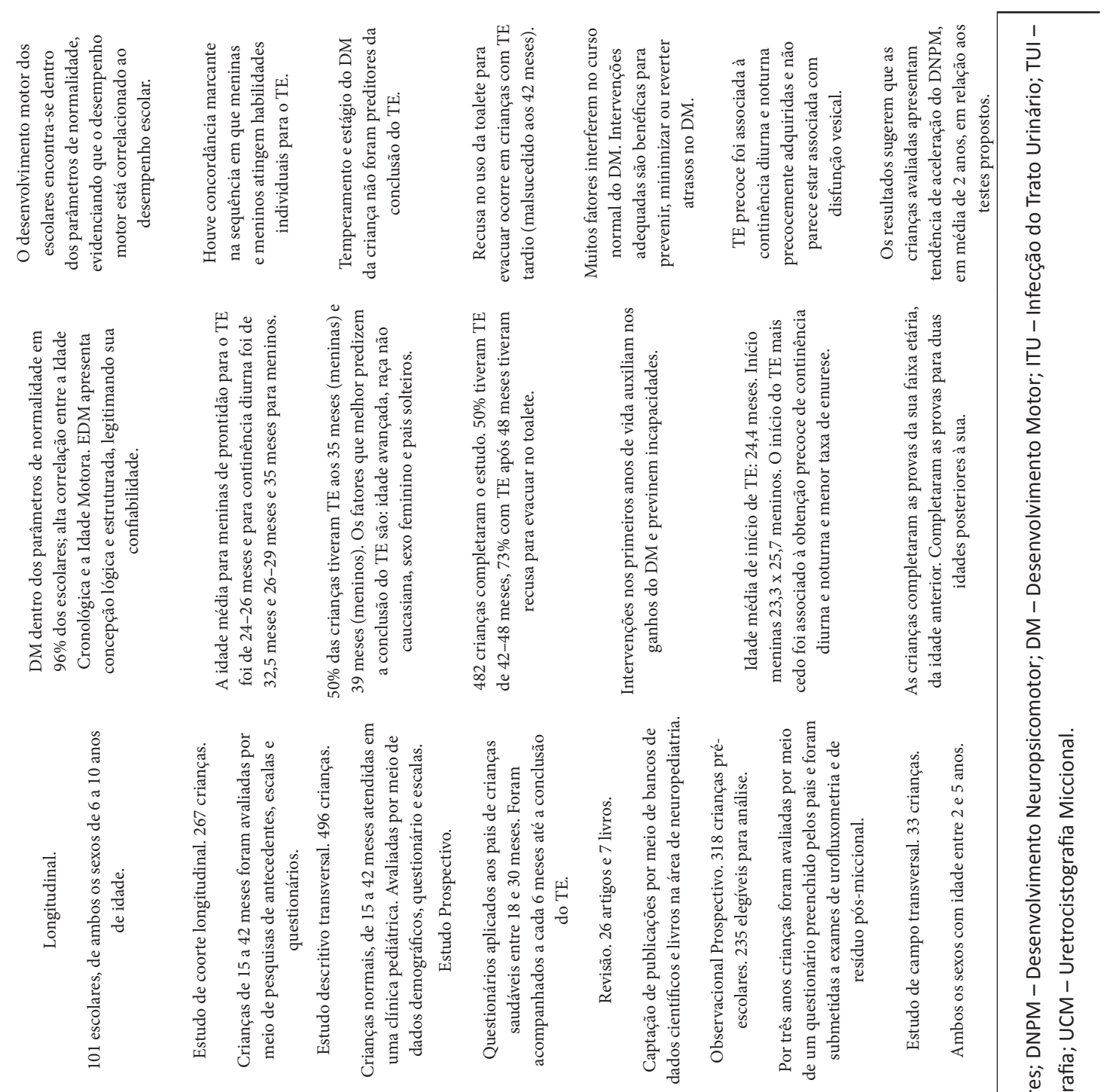

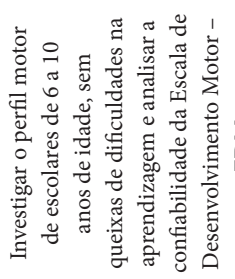

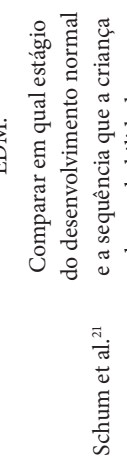

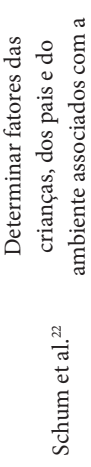
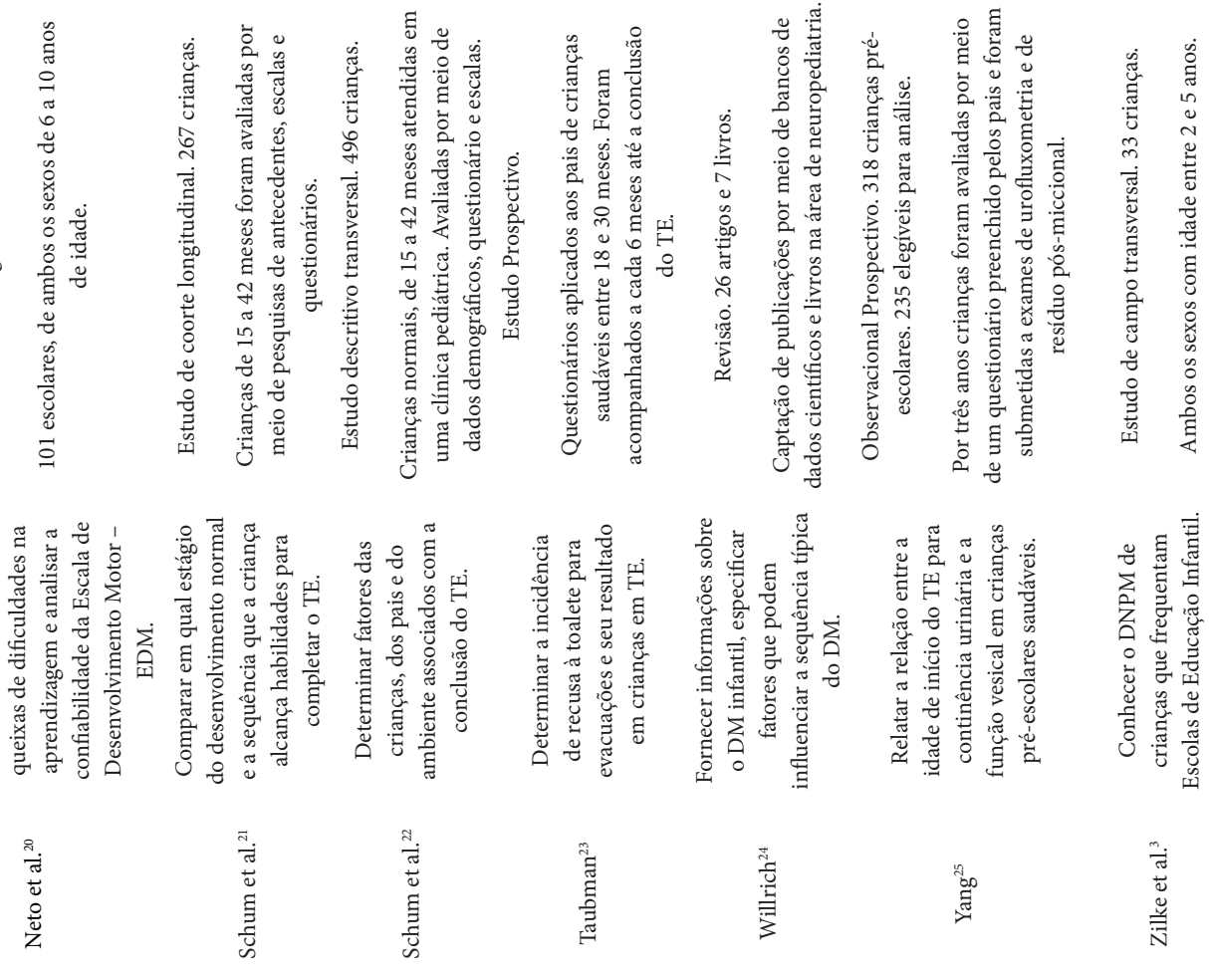

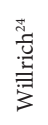

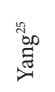

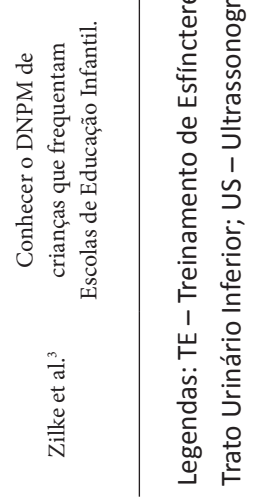




\section{Discussão}

Caetano et al. ${ }^{10}$ analisaram as alterações no desenvolvimento motor de crianças na idade de 3 a 7 anos de ambos os sexos por meio da Escala de Desenvolvimento Motor $(\mathrm{EDM})$ e concluíram que, no decorrer da vida, as habilidades e o desenvolvimento motor são mantidas ou melhoradas por meio de ajustes, compensações ou mudanças que ocorrem continuamente pela interação entre as exigências físicas, mecânicas, biológicas e ambientais. Caracteriza-se, portanto, como um processo dinâmico observado principalmente na infância, na idade pré-escolar, que é uma fase de aperfeiçoamento das habilidades motoras e mudanças comportamentais.

Existem inúmeros testes e escalas para a avaliação do desenvolvimento motor de uma criança, no entanto, quase nenhum desses instrumentos consegue englobar completamente todos os aspectos do desenvolvimento. Devido a esse fato, Neto et al., ${ }^{20}$ em seu estudo, propuseram analisar a confiabilidade da Escala de Desenvolvimento Motor - EDM, demonstrando que o padrão de desenvolvimento motor (DM) encontrado na pesquisa presume que o componente genético é o fator influenciador no processo de evolução humana, podendo outros fatores influenciar no desenvolvimento motor, principalmente variáveis do ambiente e, quando relacionada com a idade cronológica, comprova que as tarefas propostas para cada idade na escala refletem as mudanças esperadas para o DM. Para eles, a utilização desse instrumento de avaliação oportunizará a identificação de componentes do desenvolvimento motor que necessitam de aten- ção especial. Porém, a coleta foi realizada com uma população de amostra composta por crianças de 6 a 10 anos de idade.

Maronesi et al. ${ }^{18}$ avaliaram o DM de uma criança de quatro anos de idade e elaboraram uma intervenção de dois meses com atividades para adquirir itens psicomotores específicos e concluíram que, após a estimulação, mudanças psicomotoras positivas aconteceram nessa criança. Corroborando com Duong et al. ${ }^{14}$ que concluíram que é possível iniciar, através de estimulação precoce e contínua, o TE com bons resultados muito cedo, aos nove meses de idade.

Duong et al. ${ }^{14}$ compararam crianças do Vietnã e da Suíça nos seus primeiros anos de vida e concluíram que o TE realizado diariamente e precocemente ( $89 \%$ das crianças estudadas no Vietnã iniciaram o treino aos seis meses de idade) afeta positivamente a capacidade de esvaziamento da bexiga, em que nenhuma urina residual foi encontrada com a idade de 9 meses, de modo diferente das crianças suecas, em que apenas $5 \%$ iniciaram o treinamento aos 24 meses e somente aos 36 meses observou-se esvaziamento total da bexiga, sendo apoiados por Benjasuwantep e Ruangdaraganon, ${ }^{9}$ que viram que cerca de $50 \%$ das crianças tailandesas tiveram TE bem-sucedido aos 12 meses.

Hellström e Sillén ${ }^{15}$ afirmam que o TE precoce é vantajoso na disfunção vesicoureteral e diminui o risco de infecção urinária, sendo confirmado por Da Fonseca et al., ${ }^{12}$ que concluíram que o TE antes dos 24 meses não foi associad à síndrome das eliminações e por Yang et al. ${ }^{25}$ que relataram que o TE não parece estar associado à disfunção da bexiga. Entretanto, Hodges et al ${ }^{17}$ afirmaram 
que o TE antes dos 24 meses está associado com disfunção miccional.

Em outro estudo, Duong et al. ${ }^{26}$ reforçam que a incoordenação entre o esfíncter e o detrusor parece ter desaparecido aos 9 meses de idade em bebês que são treinados mais cedo, sugerindo que o treinamento é benéfico em crianças pequenas com infecções do trato urinário ou cicatrizes renais. Entretanto, em um grande estudo de corte realizado por Chen et al., ${ }^{11}$ apesar de não ter sido relatado associação entre disfunções do trato urinário e a idade do treinamento esfincteriano, a primeira infecção do trato urinário foi correlacionada com a idade precoce de TE, especialmente em meninas.

Em contrapartida Blum et al., ${ }^{27}$ em um estudo longitudinal, descobriram que a iniciação do TE na idade avançada era acompanhada de constipação e, posteriormente, recusa do uso do toalete ao evacuar, concordando com o estudo de Hodges et al., ${ }^{17}$ que relataram que o TE após 36 meses está associado com constipação e disfunção miccional.

Recomendamos conduzir novos estudos para a constatação e comparação de distúrbios do trato urinário em início precoce e tardio do TE em crianças, com um acompanhamento posterior mais prolongado para a detecção de distúrbios posteriores.

No presente estudo foi observado que países do continente asiático consideram uma idade adequada para a conclusão do TE até os 12 meses, e países das Américas e da Europa consideram precoce o TE antes dos 24 meses. O termo precoce foi utilizado em uma variação de 12 a 24 meses. Sugere-se, portanto, identificar, a fim de unificar na literatura global, o que é precoce, em termos de TE.

Caetano et al. ${ }^{10}$ objetivaram em seus estudos avaliar o desenvolvimento motor de pré-escolares, determinando a idade motora por meio da Escala de Desenvolvimento Motor de Rosa Neto, composta por testes para avaliar o desenvolvimento motor de crianças de 2 a 11 anos de idade, de acordo com sua idade cronológica e, concluíram que mudanças marcantes no processamento de informação estavam correlacionadas com a idade, ou que, com a idade, toda criança sem deficiência alcança melhoras quantitativas e qualitativas em seu desenvolvimento, sendo que o início do desenvolvimento motor não se deve apenas à maturação neurológica, mas também a um sistema auto-organizado que envolve a tare$\mathrm{fa}, \mathrm{o}$ ambiente e o indivíduo.

E, de acordo com esse achado, Sillén ${ }^{28}$ sugeriu: "a bexiga neonatal é regulada por vias neuronais com conexões ao córtex cerebral no período neonatal, o que é contrário ao conceito anterior de micção como um evento automático em resposta a um volume constante na bexiga. Essa teoria não significa que a micção seja consciente ou voluntária nessa faixa etária, mas apenas que o reflexo da micção perturba o neonato. Novas características incluem o conceito de descoordenação fisiológica, como a hiperatividade do detrusor vista como baixa capacidade da bexiga e altas pressões miccionais no período neonatal".

Idade é algo subjetivo, de modo que as crianças amadurecem em tempos distintos, mesmo dentro do padrão de normalidade. Por esse fato, o tempo de controle dos esfíncteres varia, em janelas de, por exemplo, 
dois anos (controle miccional noturno adquirido entre quatro a seis anos de idade), ${ }^{6}$ não sendo o tempo, portanto, um marcador acurado. Entretanto, Schum et al. ${ }^{21}$ descreveram em seu estudo utilizando escala de DM que houve uma concordância marcante entre as meninas e os meninos na ordem em que as 26 habilidades comuns a ambos os sexos são atingidas antes do início do TE, confirmando a Teoria dos Sistemas Dinâmicos e a sequência no desenvolvimento da criança. Nesse ínterim, o DNPM e seus marcadores específicos podem servir de variável importante para uma marcação mais acurada, ou ao menos vir a somar insights no desenvolvimento em paralelos dos controles motor e miccional. Ao considerar-se ainda o controle miccional dependente de musculatura estriada esquelética, como o rabdoesfíncter, ${ }^{29}$ parece logicamente provável que o controle esfincteriano esteja diretamente correlacionado ao desenvolvimento motor, podendo ser alcançado e determinado a partir de certa idade motora da criança.

Portanto, sugerimos estudos transversais, longitudinais e de coorte prospectivo correlacionando o DNPM, por meio de escalas validadas, com o controle de esfíncteres.

Visto que, conforme exposto, tanto o desenvolvimento motor como o TE podem ser alcançados por estimulação precoce e contínua, também sugerimos novos estudos com estímulos precoces ao controle de esfíncteres e suas consequências.

Tendo em vista o estudo de Soares et al. ${ }^{30}$ sobre as oportunidades de estimulação motora em ambientes domiciliares, o qual encontrou carência na provisão de materiais que promovem a estimulação do desenvolvimento da motricidade infantil, concorda- mos com Van Nunen et al., ${ }^{31}$ que concluíram que os pais devem ser informados sobre as possíveis consequências negativas do TE em um período impróprio para a criança, e que devem ser auxiliados nesse processo, necessitando de mais apoio e informação.

\section{CONSIDERAÇões FINAIS}

Notamos que estudos que correlacionem o DNPM e a continência urinária ainda são escassos, sendo a maioria dos estudos relacionados ao DM infantil, sem correlações com seu desenvolvimento ou maturação neurológica miccional, o que dificulta termos dados fidedignos para afirmar que a criança que apresenta algumas habilidades motoras, mesmo que não esteja em sua idade cronológica, pode ou não iniciar o TE.

Quando os estudos foram realizados com as crianças que ainda estavam em treinamento ou quando os estudos foram feitos com as crianças que já haviam sido desfraldadas, seja ele precoce ou tardio, não foi levado em consideração ou não foi o objetivo do estudo pontuar as habilidades motoras e a idade cronológica em que a criança se encontrava no período em que estava sendo desfraldada.

Entretanto, a maior parte dos estudos sugere que o TE precoce (de 12 a 24 meses) não tem correlação com disfunção miccional ou síndrome das eliminações, diferente do início tardio (acima de 36 meses). Contudo, os artigos analisados que falam sobre ou defendem o TE precoce ou tardio não acompanharam as crianças pós- desfralde tempo suficiente para observarem possíveis alterações decorrentes do treinamento.

O DNPM e seus marcadores específicos podem servir de variável importante para 
uma marcação mais acurada, ou ao menos vir a somar insights no desenvolvimento em paralelos dos controles motor e miccional. Ao considerar-se ainda o controle miccional dependente de musculatura estriada esquelética, como o rabdoesfíncter, parece logicamente provável que o controle esfincteriano esteja diretamente correlacionado ao desenvolvimento motor.
Ante o exposto, o presente estudo sugere novas investigações para correlacionar o desenvolvimento neuropsicomotor da criança com o estado da continência urinária, visando iniciar a discussão quanto à definição de marcadores mais precisos de desenvolvimento para uma orientação mais adequada quanto ao início do TE, evitando distúrbios miccionais decorrentes do início precoce ou tardio do processo de desfralde.

\section{REFERÊNCIAS}

1. Mendes RP. Atualização em Nefrologia Pediátrica: Distúrbios Funcionais da Micção da Infância. J Bras Nefrol. 2000; 22 (2) 95-102.

2. Braga AKP, Rodovalho JC, Formiga, CKMR. Evolução do crescimento e desenvolvimento neuropsicomotor de crianças pré-escolares de zero a dois anos do município de Goiânia (GO). Rev. bras. crescimento desenvolv. hum. 2011; 21 (20) 230-239.

3. Zilke R., Bonamingo Ecb, Winkelmann ER, Desenvolvimento neuropsicomotor de Crianças de 2 a 5 anos que frequentam escolas de educação infantil. Fisioter. Mov. 2009, v. 22, n. 3.

4. Souza Sandra Coenga de, Leone Claudio, Takano Olga Akiko, Moratelli Hélio Borba. Desenvolvimento de pré-escolares na educação infantil em Cuiabá, Mato Grosso, Brasil. Cad. Saúde Pública. 2008; 24 (8): 1.917-1.926.

5. Kranz S., Findeis JL, Shrestha SS. Uso do Índice de Qualidade da Dieta Infantil Revisado para avaliar a dieta alimentar de pré-escolares, seus preditores sociodemográficos e sua associação com peso corporal. J. Pediatr. 2008; 8 (1).

6. Koppen IJ, von Gontard A., Chase J., Cooper CS, Rittig CS, Bauer SB, Homsy Y., Yang SS, Benninga MA (2016) Management of functional nonretentive fecal incontinence in children: recommendations from the International Children's Continence Society. J Pediatr Urol 12: 56-64.

7. Bakker E., Van Gool J., Wyndaele Jj. Results of a questionnaire evaluating different aspects of personal and familial situation, and the methods of potty-training in two groups of children with a different outcome of bladder control. Scand J Urol Nephrol. 2001 Oct.; 35 (5): 370-6.

8. Blum NJ, Taubman B., Nemeth N. Relationship between age at initiation of toilet training and duration of training: a prospective study. Pediatrics. 2003 Apr.; 111 (4 Pt 1): 810-4.

9. Benjasuwantep B., Ruangdaraganon N. Infant toilet training in Thailand: starting and completion age and factors determining them. J Med Assoc Thai. 2011 Dec.; 94 (12): 1.441-6.

10. Caetano MJD, Silveira CRA, Gobbi LTB. Desenvolvimento motor de pré-escolares no intervalo de 13 meses. Rev. Bras. Cineantropom. Desempenho. Hum. 2005; 7 (2): 05-13.

11. Chen JJ, Ahn HJ, Steinhardt GF. Is age at toilet training associated with the presence of vesicoureteral reflux or the occurrence of urinary tract infection? J Urol. 2009; 182(1):268-71.

12. da Fonseca EM, ${ }^{1}$ Santana PG, Gomes FA, Bastos MD. Dysfunction elimination syndrome: is age at toilet training a determinant? J Pediatr Urol. 2011 Jun.; 7 (3): 332-5.

13. Duong TH, Jansson UB, Hellström AL. Vietnamese mother's experiences with potty training procedure for children from birth to 2 years of age. J Pediatr Urol. 2013 Dec.; 9 (6 Pt A): 808-14. 
14. Duong TH, Jansson UB, Holmdahl G., Sillén U., Hellström AL. Urinary bladder control during the first 3 years of life in healthy children in Vietnam - a comparison study with swedish children. J Pediatr Urol. 2013 Dec.; 9 (6 Pt A): 700-6.

15. Hellström AL, sillén $U$. Early potty training advantageous in bladder dysfunction. Decreases the risk of urinary infection. Lakartidningen. Lakartidningen. 2001 Jul 11; 98 (28-29): 3.216-9.

16. Hooman N., Safaii A., Alavijeh ZA. Toilet Training in Iranian Children: A Cross-Sectional Study. Iran J Pediatr. 2013; 23 (2): 154-158.

17. Hodges SJ, Richards KA, Gorbachinsky I., Krane S. The association of age of toilet training and dysfunctional voiding. Res rep urol. 2014; 6: 127-130.

18. Maronesi LC, Figueiredo MO, Santos E., Gonçalves SMM, CAMPOS SDF. Análise de uma intervenção dirigida ao desenvolvimento da coordenação motora fina, global e do equilíbrio. Cad. Ter. Ocup. 2015; 23 (2): 273-284.

19. Mota DM, Barros AJ. Toilet training: situation at 2 years of age in a birth cohort. J Pediatr (Rio J), v. 84, n. 5, 2008.

20. Neto RF, Santos APM, Xavier RFC, Amaro KN. A Importância da avaliação motora em escolares: análise da confiabilidade da Escala de Desenvolvimento Motor. Rev Bras Cineantropom Desempenho Hum. 2010; 12 (6): 422-427.

21. Schum TR, Kolb TM, McAuliffe TL, Simms MD, Underhill RL, Lewis M. Sequential acquisition of toilet-training skills: a descriptive study of gender and age differences in normal children. Pediatrics. 2002 Mar.; 109 (3): E48.

22. Schum TR, McAuliffe TL, Simms MD, Walter JA, Lewis M., Pupp R. Factors associated with toilet training in the 1990s. Ambul Pediatr. 2001 Mar./Apr; 1 (2): 79-86.

23. Taubman, B. Toilet training and toileting refusal for stool only: a prospective study. Pediatrics. 1997; 99: 54-8.

24. Willrich A., Azevedo CCF, Fernanses JO. Desenvolvimento motor na infância: influência dos fatores de risco e programas de intervenção. Rev Neurocienc. 2009; 17 (1): 51-56.

25. Yang SS, ${ }^{\text {Zhao LL }}$, Chang SJ. Early initiation of toilet training for urine was associated with early urinary continence and does not appear to be associated with bladder dysfunction. Neurourology and Urodynamics. 2011; 30: 1.253-1.257.

26. Duong TH, Jansson UB, Holmdahl G., Sillén U., Hellstrom AL. Development of bladder control in the first year of life in children who are potty trained early. J Pediatr Urol. 2010; 6 (5): 501-5.

27. Blum NJ, ${ }^{\text {Taubman B. }}$, Nemeth N. Why is toilet training occurring at older ages? A study of factors associated with later training. J Pediatr. 2004; 145 (1): 107-11.

28. Sillén, U. Bladder function in healthy neonates and its development during infancy. J Urol. 2001 Dec.; 166 (6): 2.376-81

29. Bø K., Berghmans B., Mørkved S., VAN KAMPEN M. Evidence-Based Physical Therapy for the Pelvic Floor: Bridging Science and Clinical Practice. 2. ed. London: Churchill Livingstone, 446, p. 2.015.

30. Soares ES, Flores FS, Katzer JI, Valentini NC, Corazza ST, Copetti F. Análise das oportunidades de estimulação motora em ambientes domiciliares na região central do Rio Grande do Sul. Rev. bras. educ. fís. esporte. 2015; 29 (2): 279-288.

31. Van Nunen K., Kaerts N., Wyndaele JJ, Vermandel A., Hal GV. Parent's views on toilet training (TT): A quantitative study to identify the beliefs and attitudes of parents concerning TT. J Child Health Care. 2015 Jun.; 19 (2): 265-74. 


\section{DADOS DOS AUTORES}

\section{Bianca da Silva Januário Abdalla}

Pós-graduanda, curso fisioterapia pélvica/uroginecologia funcional. Faculdade Inspirar. Curitib/ PR - Brasil.biagaquaps@hotmail.com

\section{Dhaiany Gomes Dourado}

Pós-graduanda, curso fisioterapia pélvica/uroginecologia funcional. Faculdade Inspirar. Cuiabá/MT

- Brasil.dhaiany_dourado@hotmail.com

\section{ERICA fEIo CARNeiro Nunes}

Doutora em Ciências da Reabilitação. Professora da Universidade do Estado do Pará. Belém/PA Brasil.erica@perineo.net

\section{Gustavo Fernando Sutter LAtorre}

Fisioterapeuta pélvico, Mestre em Fisioterapia. Rede Perineo.net. Florianópolis, SC - Brasil. gustavo@perineo.net

Submetido em: 26-12-2018

Aceito em: 22-6-2020 